Article

\title{
Investigation of Intestinal Microbiota and Fecal Calprotectin in Non-Toxigenic and Toxigenic Clostridioides difficile Colonization and Infection
}

\author{
Sung-Hee Han ${ }^{1}$, Joowon $\mathrm{Yi}^{2}$, Ji-Hoon $\mathrm{Kim}^{3}$ and Hee-Won Moon ${ }^{4, *}$ \\ 1 BioCore Co. Ltd., Biotechnology, Yongin 64844, Korea; hansungh@bio-core.com \\ 2 Samkwang Medical Laboratories, Seoul 06742, Korea; caod.alt@smlab.co.kr \\ 3 Advanced BioVision Inc., Incheon 21999, Korea; patriet80@gmail.com \\ 4 Department of Laboratory Medicine, Konkuk University School of Medicine, Seoul 05030, Korea \\ * Correspondence: hannasis@kuh.ac.kr; Tel.: +82-2-2030-5583; Fax: +82-2-2030-5587
}

Received: 26 May 2020; Accepted: 9 June 2020; Published: 11 June 2020

\begin{abstract}
In this study, we aimed to evaluate the composition of the intestinal microbiota and level of fecal calprotectin in Clostridioides difficile-colonized patients. We included 102 C. difficile non-colonized (group I), 93 C. difficile colonized subjects (group II), and 89 diarrhea patients with C. difficile (group III). Chao1 index for alpha diversity and principal coordinate analysis was performed for beta diversity using QIIME. The mean relative abundance in each group was compared at the phylum and genus levels. Fecal calprotectin was measured using EliA calprotectin (Thermo Fisher Scientific). Group II showed significantly lower levels of Sutterella, Blautia, Ruminococcus, Faecalibacterium, Bilophila, and Ruminococcaceae and higher levels of Enterobacteriaceae compared to group I ( $p=0.012,0.003,0.002$, $0.001,0.027,0.022$, and 0.036 , respectively). Toxigenic $C$. difficile colonized subjects showed significantly lower levels of Prevotella, Phascolarctobacterium, Succinivibrio, Blautia, and higher levels of Bacteroides. The level of fecal calprotectin in group III was significantly higher than those in group I and group II ( $p<0.001$ for both). These data could be valuable in understanding $C$. difficile colonization process and the microbiota and inflammatory markers could be further studied to differentiate colonization from CDI.
\end{abstract}

Keywords: Clostridioides difficile infection; colonization; microbiota; calprotectin

\section{Introduction}

Clostridioides difficile, previously known as Clostridium difficile, is an anaerobic Gram-positive, spore-forming bacterium and a common cause of antibiotic-associated nosocomial diarrhea in the industrialized world [1,2]. After the use of antibiotics, microbiota-mediated colonization resistance is decreased partly by the reduced diversity of the intestinal microbiota [3-7]. Decreased diversity and alteration of the intestinal microbiota in C. difficile infection (CDI) has been shown in previous studies using various techniques from culture-based methods to high throughput sequencing [5,7-11]. The current standard diagnosis of CDI is made from the presence of toxigenic $C$. difficile in patients with significant diarrhea, defined as unexplained and new-onset ( $\geq 3$ unformed stools in $24 \mathrm{~h}$ ) [12]. Unfortunately, current diagnostic tests for CDI detect toxigenic C. difficile or its toxins or toxin gene. Many assays cannot differentiate between colonization and infection. This causes a false classification of $C$. difficile-colonized patients with diarrhea as CDI $[13,14]$. Meanwhile, direct fecal biomarkers related to intestinal inflammation, such as calprotectin, were shown to significantly increase in CDI and were proposed as severity markers $[15,16]$. Although recent guidelines do not recommend inflammatory markers for diagnosis or prognosis determination in CDI due to insufficient data, the research is 
ongoing and could be evaluated for differentiation between colonized patients and infection [13,17]. To the best of our knowledge, data for the human microbiota composition and inflammatory status in C. difficile-colonized subjects is sparse and there are no data on the comparison of toxigenic and non-toxigenic $C$. difficile colonized subjects.

The present study aimed to evaluate the composition of the intestinal microbiota and inflammatory status in C. difficile colonized subjects and compare them with those in non-colonized control and patients with significant diarrhea. We also compared the differences between toxigenic and non-toxigenic C. difficile colonized subjects.

\section{Materials and Methods}

\subsection{Clinical Samples}

This study was approved by the Institutional Review Board of the Konkuk University Medical Center, Seoul, Korea (a tertiary referral hospital). In addition to this, all methods were performed in accordance with the relevant guidelines and regulations. From March 2018 to June 2019, we screened for $C$. difficile colonization in 812 residual fecal samples from individuals who visited our center for a general health examination. Korean adults ( $>30$ years old) were included and subjects with obesity (body mass index $>25$ ), other ethnicity or history of recent hospitalization were excluded. C. difficile colonization was firstly assessed by glutamate dehydrogenase (GDH) testing using VIDAS C. difficile GDH on the VIDAS instrument (bioMérieux, Marcy-l'Etoile, France) according to the manufacturer's instructions. Among the screened samples, 93 (11.4\%) were GDH-positive. We defined "C. difficile colonization" as positive GDH in the absence of CDI symptoms [14]. We included randomly selected 102 GDH-negative samples (group I, C. difficile non-colonized) and 93 GDH-positive samples (group II, C. difficile colonized) for further study. Toxin status was determined by $t c d B$ gene real-time PCR (Xpert C. difficile system, Cepheid, Sunnyvale, CA, USA). The Xpert C. difficile is an automated, real-time multiplex PCR assay, which detects the genes for $t c d B$, binary toxin $(c d t)$, and a point mutation at position 117 associated with hypervirulent 027/NAP1/BI strains. A maximum valid $\mathrm{Ct}$ is $37 \mathrm{for} t c d B$ and $c d t$ according to the manufacturer's instructions. Among the $93 \mathrm{C}$. difficile-colonized subjects (group II), $13(14 \%)$ were $t c d B$ gene positive. In addition, we collected $89 \mathrm{GDH}$-positive samples from hospitalized patients with significant diarrhea ( $\geq 3$ unformed stools in $24 \mathrm{~h}$ ) [3,18]. They received antibiotics and most patients were from the hematologic and gastrointestinal department. We excluded the patients with laxative use in $48 \mathrm{~h}$ before sample collection to rule out other reasons for diarrhea.

They included $20 t c d B$ gene-negative and $69 t c d B$ gene-positive samples (group III, diarrhea with C. difficile). A total of 284 samples were analyzed and the characteristics of each group are summarized in Table 1. Upon reception, stool samples were stored at $-70{ }^{\circ} \mathrm{C}$ until use. This study required neither study-specific nor any other interventions and the data were analyzed anonymously. Therefore, written informed consent from the enrolled patients was waived by the ethics committee.

Table 1. Demographic information for the subject in each group.

\begin{tabular}{ccccc}
\hline Groups & Source & Number & Sex (M/F) & Age, Years (Median, IQR) \\
\hline $\begin{array}{c}\text { Group I } \\
\text { C. difficile- non colonized } \\
\text { Group II }\end{array}$ & $\begin{array}{c}\text { General } \\
\text { examination } \\
\text { General } \\
\text { C. difficile-colonized } \\
\text { Group III }\end{array}$ & 102 & $52 / 50$ & $57.0,46.8-63.3$ \\
Diarrhea with C. difficile & $\begin{array}{c}\text { Examination } \\
\text { Hospitalized } \\
\text { patients }\end{array}$ & 89 & $61 / 32$ & $54.0,47.0-63.0$ \\
\hline
\end{tabular}

IQR, interquartile values. ${ }^{*} p=0.001$ and $<0.001$ compared group I and group II, respectively. 


\subsection{Microbiome Analysis}

DNA extraction from a stool sample was performed using a QIAamp DNA stool mini kit (Qiagen, Valencia, CA, USA) and bacterial 16S rRNA genes were amplified using an Ion 16S ${ }^{\mathrm{TM}}$ Metagenomics Kit (ThermoFisher Scientific, Waltham, MA, USA) according to the manufacturer's protocol. The kit included 2 primer tubes with 3 primer sets that amplify the hypervariable regions of 16S rRNA (V2, V4, V8 and V3, V6-7, V9, respectively). After PCR amplicons were purified using Agencourt AMPure ${ }^{\circledR}$ XP beads (Beckman Coulter, Indianapolis, IN, USA), sequencing libraries were prepared using an Ion Plus Fragment Library Kit and Ion Xpress ${ }^{\mathrm{TM}}$ Barcode Adapters (ThermoFisher Scientific, Waltham, MA, USA). Prepared libraries were quantified using a High Sensitivity DNA kit on an Agilent 2100 Bioanalyzer (Agilent Technologies, Santa Clara, CA, USA). Template preparation and sequencing were performed using the Ion Chef ${ }^{\mathrm{TM}}$ System and Ion S5 ${ }^{\mathrm{TM}}$ XL system with Ion $530^{\mathrm{TM}}$ Chip Kit (ThermoFisher Scientific, Waltham, MA, USA). After filtering out low quality and polyclonal reads, and trimming any adaptor sequences at the $3^{\prime}$ end, the sequencing data were exported as FASTQ files and were processed using the Quantitative Insights into Microbial Ecology (QIIME) pipeline 1.9.1 [19]. After quality filtering, 26,634,236 sequences were obtained, with a mean of 89,165 sequences per sample (min: 16,585, max: 335,275). Operational taxonomic units (OTUs) were clustered based on $97 \%$ sequence similarity with at least 10 identical sequences and assigned against the curated Greengenes v.13.8 reference database at the QIIME web site (http://qiime.org/home_static/dataFiles.html).

Alpha and beta diversity measures were calculated by QIIME [20]. Alpha diversity assessment was based on observed OTUs, including unidentified OTUs, and Chao1 index. Microbial diversity was visualized using principal coordinate analysis ( $\mathrm{PCOA})$ and microbial diversity between samples was assessed by qualitative (unweighted UniFrac) and quantitative (weighted UniFrac) distances. The mean relative abundance (percentage among all reads) in each group was compared at the phylum and genus levels.

\subsection{Calprotectin Measurement}

After thawing, fecal samples were extracted using EliA Stool Extraction Kit (Thermo Fisher Scientific, Waltham, MA, USA). The extraction tubes were pre-filled with $750 \mu \mathrm{L}$ of EliA Calprotectin Extraction Buffer. Fecal calprotectin was measured using EliA calprotectin on the Phadia 250 system (Thermo Fisher Scientific, Waltham, MA, USA). The wells of the EliA Calprotectin were coated with monoclonal antibodies against calprotectin, and the calprotectin levels were quantified by fluoroenzyme immunoassay. Phadia 250 measures calprotectin concentrations in $\mathrm{ng} / \mathrm{mL}$, and by using a conversion factor given by the lot-specific code of the EliA Calprotectin Well, the results were automatically converted to $\mathrm{mg} / \mathrm{kg}$.

\subsection{Statistical Analysis}

The difference between the continuous variables was analyzed using Student's $t$-test or the Mann-Whitney $U$ test, and that between categorical variables was analyzed using the chi-squared test, Fisher's exact test, or the McNemar test. The Kruskal-Wallis test and one-way analysis of variance (ANOVA) were used to assess the differences between groups. Permutational multivariate analysis of variance (PERMANOVA) between groups was performed using QIIME. Statistical analysis was performed using MedCalc Statistical Software (v. 15.8, MedCalc Software, Mariakerke, Belgium) and IBM SPSS Statistics 22.0 (IBM Corporation, Armonk, NY, USA). $p$ values less than 0.05 were considered statistically significant.

\section{Results}

\subsection{Comparison of Alpha Diversity of the Intestinal Microbiota Across Different Groups}

We evaluated the differences in alpha diversity between group I (C. difficile-non colonized), group II (C. difficile-colonized), and group III (diarrhea with C. difficile). The distribution of the Chao1 indexes in 
each group is presented in Figure 1. The median Chao1 index of group III [median, interquartile range (IQR), 102.3, 85.7-127.1] was significantly lower than that of group I and group II (120.8, 105.7-136.9 and $119.5,109.2-136.3, p<0.001$ for both). The median Chao1 index between group I and group II was not significantly different $(p=0.797)$. The median Chao1 index between subjects with non-toxigenic and toxigenic C. difficile was not significantly different in group II and III ( $p=0.287$ and 0.988 , respectively).
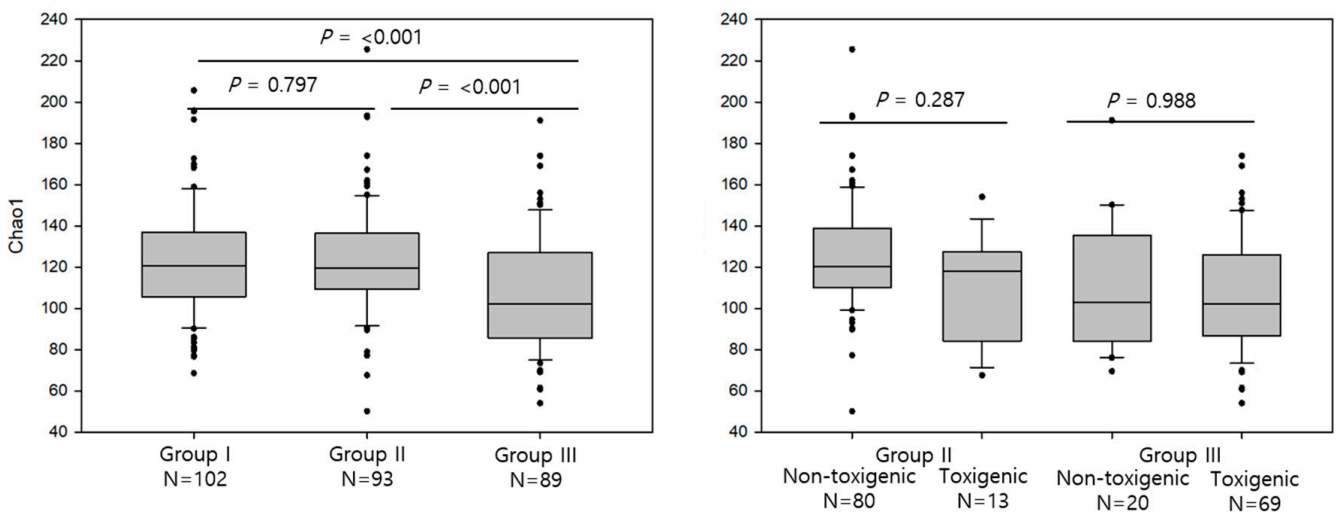

Figure 1. Alpha diversity (Chao1 index) in group I (C. difficile non-colonized), group II (C. difficile colonized), and group III (diarrhea with $C$. difficile) are shown in left and the comparisons between non-toxigenic and toxigenic $C$. difficile within group II and group III are shown in right. Each $p$-value (I vs. III, I vs. II, II vs. III, and non-toxigenic vs. toxigenic C. difficile) was presented. The box plot shows the median and interquartile values. The whiskers indicate 1.5 times the interquartile range above and below the 75 th and 25 th percentiles. The circles indicate the outliers.

\subsection{Comparison of Beta Diversity of the Intestinal Microbiota Across Different Groups}

PCoA using unweighted and weighted UniFrac matrix was performed to evaluate the beta diversity between each group. In unweighted UniFrac analysis, group I vs. III and group II vs. III clustered separately (both $p=0.001$ by PERMANOVA), while group I and II could not be separated ( $p=0.057$ by PERMANOVA). In weighted UniFrac analysis, all three groups clustered separately ( $p=0.017,0.001$, and 0.001 for group I vs. II, group I vs. III, and group II vs. III, respectively) (Figure 2). In group II, subjects with non-toxigenic and toxigenic $C$. difficile clustered separately both in unweighted and weighted UniFrac matrix (both $p=0.001$ ). In group III, subjects with non-toxigenic and toxigenic C. difficile clustered separately only in unweighted UniFrac matrix ( $p=0.001$ in unweighted and 0.721 in weighted UniFrac matrix).
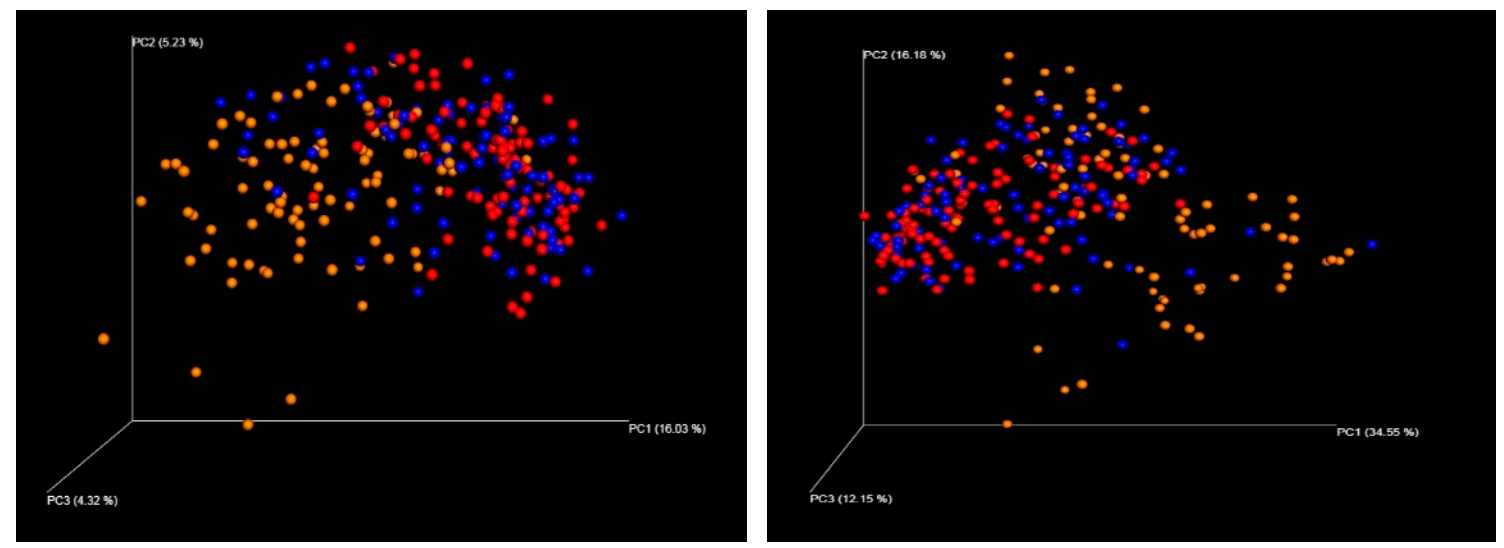

Figure 2. Evaluation of beta diversity in group I (red), II (blue), and III (orange). Principal coordinate analysis (PCoA) was performed using unweighted (left) and weighted (right) UniFrac distances of $16 \mathrm{~S}$ rRNA gene sequences. Each axis represents inter-sample variation. 


\subsection{Comparison of Mean Relative Abundance in Each Group at the Phylum Level}

The comparison of mean relative abundance in groups I, II, and III at the phylum level is shown in Table 2. Group III showed significantly lower levels of Bacteroidetes $(p<0.001$ and 0.001 compared with groups I and II, respectively) and significantly higher levels of Proteobacteria compared to group I $(p<0.001)$. Comparing subjects with non-toxigenic and toxigenic strains in group II, those with toxigenic C. difficile colonized subjects showed significantly lower levels of Firmicutes $(p<0.001)$. In group III, there was no significant difference in composition between patients infected with non-toxigenic and toxigenic C. difficile strains (Table 3).

Table 2. Comparison of the mean relative abundance (\%) at phylum level in C. difficile-non colonized (group I), colonized (group II), and diarrhea with C. difficile (group III).

\begin{tabular}{ccccccc}
\hline \multirow{2}{*}{ Phylum } & Group I & Group II & Group III & \multicolumn{3}{c}{$\boldsymbol{p}$} \\
\cline { 5 - 7 } & $(\boldsymbol{n}=\mathbf{1 0 2})$ & $(\boldsymbol{n}=\mathbf{9 3})$ & $(\boldsymbol{n}=\mathbf{8 9 )}$ & $\mathbf{I}$ vs. II & I vs. III & II vs. III \\
\hline Bacteroidetes & 43.85 & 43.22 & 32.41 & 0.911 & $<0.001$ & 0.001 \\
Firmicutes & 33.55 & 29.25 & 32.45 & 0.065 & 0.924 & 0.542 \\
Proteobacteria & 20.91 & 25.75 & 32.67 & 0.096 & $<0.001$ & 0.077 \\
Actinobacteria & 0.98 & 0.81 & 1.1 & 0.564 & 0.952 & 0.75 \\
Fusobacteria & 0.64 & 0.89 & 0.97 & 0.753 & 0.724 & 0.987 \\
\hline
\end{tabular}

Phyla with mean relative abundance $>1.0 \%$ were described in Table 2 .

Table 3. Comparison of the mean relative abundance (\%) of the composition of phyla between non-toxigenic and toxigenic $C$. difficile in group II and group III.

\begin{tabular}{ccccccc}
\hline Phylum & \multicolumn{2}{c}{ Group II } & \multicolumn{3}{c}{ Group III } \\
Non-Toxigenic & Toxigenic & $p$ & $\begin{array}{c}\text { Non-Toxigenic } \\
(\boldsymbol{n}=\mathbf{8 0})\end{array}$ & $\begin{array}{c}(\boldsymbol{n}=\mathbf{1 3}) \\
\text { Toxigenic })\end{array}$ & $\boldsymbol{p}$ \\
\hline Bacteroidetes & 42.85 & 45.46 & 0.491 & 32.21 & 32.47 & 0.968 \\
Firmicutes & 31.15 & 17.57 & $<0.001$ & 31.62 & 32.69 & 0.865 \\
Proteobacteria & 24.63 & 32.67 & 0.137 & 34.73 & 32.08 & 0.668 \\
Acinetobacter & 0.87 & 0.44 & 0.151 & 0.48 & 1.28 & 0.371 \\
Fusobacteria & 0.42 & 3.83 & 0.059 & 0.62 & 1.08 & 0.624 \\
\hline
\end{tabular}

Phyla with mean relative abundance $>1.0 \%$ were described.

\subsection{Comparison of Mean Relative Abundance in Each Group at the Genus Level}

The comparison of mean relative abundance in groups I, II, and III at the genus level is shown in Table 4. Group III showed a significantly lower mean relative abundance of many genera (Prevotella, Lachnospira, Blautia, Ruminococcus, Phascolarctobacterium, Faecalibacterium, Bilophila, unassigned genus of Ruminococcaceae, and Lachnospiraceae) compared with those in groups I and II. Group III also showed $a$ significantly higher mean relative abundance of Parabacteroides, Serratia, Veillonella, Enterococcus, and an unassigned genus of Enterobacteriaceae. Group II showed a significantly lower abundance of Sutterella, Blautia, Ruminococcus, Faecalibacterium, Bilophila, and an unassigned genus of Ruminococcaceae and higher abundance of an unassigned genus of Enterobacteriaceae compared with those in group I $(p=0.012,0.003$, $0.002,0.001,0.027,0.022$, and 0.036, respectively). Moreover, toxigenic $C$. difficile colonized subjects showed a significantly lower abundance of Prevotella, Phascolarctobacterium, Succinivibrio, Blautia, and an unassigned genus of Ruminococcaceae, and higher abundance of Bacteroides and an unassigned genus of Enterobacteriaceae compared with non-toxigenic $C$. difficile colonized subjects $(p=0.014,<0.001,0.019$, $0.006,<0.001,0.035$, and 0.044 , respectively). In group III, there was no significant difference between patients with non-toxigenic and toxigenic C. difficile (Table 5). 
Table 4. Comparison of the mean relative abundance (\%) at genus level C. difficile-non-colonized (group I), colonized (group II), and diarrhea with C. difficile (group III).

\begin{tabular}{|c|c|c|c|c|c|c|}
\hline Genus & $\begin{array}{c}\text { Group I } \\
(n=102)\end{array}$ & $\begin{array}{c}\text { Group II } \\
(n=93)\end{array}$ & $\begin{array}{c}\text { Group III } \\
(n=89)\end{array}$ & I vs. II & $\begin{array}{c}p \\
\text { I vs. III }\end{array}$ & II vs. III \\
\hline Bacteroides & 26.68 & 26.44 & 23.48 & 0.993 & 0.448 & 0.543 \\
\hline Enterobacteriaceae * & 11.42 & 16.59 & 22.1 & 0.036 & $<0.001$ & 0.067 \\
\hline Prevotella & 10.09 & 8.94 & 1.36 & 0.756 & $<0.001$ & $<0.001$ \\
\hline Ruminococcaceae * & 8.08 & 6.13 & 1.68 & 0.022 & $<0.001$ & $<0.001$ \\
\hline Lachnospiraceae * & 7.16 & 6.06 & 3.49 & 0.118 & $<0.001$ & 0.001 \\
\hline Sutterella & 3.13 & 1.97 & 1.38 & 0.012 & 0.002 & 0.441 \\
\hline Rikenellaceae * & 1.95 & 1.58 & 1.06 & 0.35 & 0.013 & 0.215 \\
\hline Lachnospira & 1.84 & 1.36 & 0.27 & 0.055 & $<0.001$ & $<0.001$ \\
\hline Blautia & 1.62 & 1.15 & 0.5 & 0.003 & $<0.001$ & $<0.001$ \\
\hline Ruminococcus & 1.61 & 0.79 & 0.14 & 0.002 & $<0.001$ & $<0.001$ \\
\hline Phascolarctobacterium & 1.54 & 1.76 & 0.45 & 0.87 & $<0.001$ & 0.008 \\
\hline Coprococcus & 1.25 & 1.05 & 0.2 & 0.514 & $<0.001$ & 0.514 \\
\hline Parabacteroides & 1.42 & 1.92 & 4.23 & 0.215 & 0.001 & 0.011 \\
\hline Serratia & 1.34 & 1.5 & 3.79 & 0.842 & $<0.001$ & $<0.001$ \\
\hline Faecalibacterium & 1.25 & 0.88 & 0.38 & 0.001 & $<0.001$ & 0.002 \\
\hline Oscillospira & 1.08 & 0.98 & 1.41 & 0.574 & 0.562 & 0.379 \\
\hline Bilophila & 1.08 & 0.69 & 0.3 & 0.027 & $<0.001$ & 0.024 \\
\hline Veillonella & 0.3 & 0.45 & 2.25 & 0.51 & 0.003 & 0.007 \\
\hline Succinivibrio & 0.3 & 1.12 & 0 & 0.248 & 0.341 & 0.05 \\
\hline Lactobacillus & 0.16 & 0.46 & 1.41 & 0.33 & 0.113 & 0.185 \\
\hline Clostridium & 0.11 & 0.13 & 1.34 & 0.835 & 0.264 & 0.276 \\
\hline Eubacterium & 0.08 & 0.21 & 1.41 & 0.822 & 0.291 & 0.239 \\
\hline Enterococcus & 0.03 & 0.46 & 9.13 & 0.542 & $<0.001$ & $<0.001$ \\
\hline
\end{tabular}

Only genera with mean relative abundance $>1 \%$ were described in Table $4 .{ }^{*}$ unspecified to a specific genus within this family.

Table 5. Comparison of the mean relative abundance (\%) of the composition of genera between non-toxigenic and toxigenic $C$. difficile in groups II and III.

\begin{tabular}{|c|c|c|c|c|c|c|}
\hline \multirow[b]{2}{*}{ Genus } & \multicolumn{2}{|c|}{ Group II } & \multicolumn{4}{|c|}{ Group III } \\
\hline & $\begin{array}{l}\text { Non-Toxigenic } \\
\quad(n=80)\end{array}$ & $\begin{array}{l}\text { Toxigenic } \\
(n=13)\end{array}$ & $p$ & $\begin{array}{l}\text { Non-Toxigenic } \\
\quad(n=20)\end{array}$ & $\begin{array}{l}\text { Toxigenic } \\
(n=69)\end{array}$ & $p$ \\
\hline Bacteroides & 25.03 & 35.11 & 0.035 & 19.23 & 24.71 & 0.312 \\
\hline Enterobacteriaceae * & 15.26 & 24.75 & 0.044 & 25.77 & 21.03 & 0.368 \\
\hline Prevotella & 9.83 & 3.47 & 0.014 & 0.7 & 1.55 & 0.445 \\
\hline Ruminococcaceae * & 6.85 & 1.73 & $<0.001$ & 2.62 & 1.4 & 0.22 \\
\hline Lachnospiraceae * & 6.36 & 4.24 & 0.073 & 2.65 & 3.73 & 0.423 \\
\hline Sutterella & 2.1 & 1.19 & 0.05 & 0.74 & 1.56 & 0.407 \\
\hline Phascolarctobacterium & 2.02 & 0.21 & $<0.001$ & 0.54 & 0.46 & 0.713 \\
\hline Rikenellaceae * & 1.67 & 0.98 & 0.204 & 1.37 & 0.97 & 0.501 \\
\hline Parabacteroides & 1.66 & 3.52 & 0.265 & 6.93 & 3.45 & 0.166 \\
\hline Lachnospira & 1.43 & 0.94 & 0.25 & 0.49 & 0.2 & 0.395 \\
\hline Serratia & 1.41 & 2.08 & 0.32 & 3.67 & 3.81 & 0.902 \\
\hline Succinivibrio & 1.3 & 0 & 0.019 & 0 & 0 & 0.33 \\
\hline Ruminococcus & 1.3 & 1.59 & 0.127 & 0.16 & 0.14 & 0.753 \\
\hline Blautia & 1.26 & 0.45 & 0.006 & 0.3 & 0.56 & 0.297 \\
\hline Coprococcus & 1.12 & 0.63 & 0.199 & 0.12 & 0.22 & 0.462 \\
\hline Oscillospira & 0.91 & 1.42 & 0.158 & 1.53 & 1.38 & 0.84 \\
\hline Pseudomonas & 0.43 & 1.57 & 0.161 & 0.71 & 0.6 & 0.865 \\
\hline Fusobacteriim & 0.4 & 3.66 & 0.062 & 0.56 & 0.82 & 0.702 \\
\hline Veillonella & 0.35 & 1.1 & 0.102 & 0.39 & 2.79 & 0.002 \\
\hline Enterococcus & 0.5 & 0.19 & 0.793 & 15.23 & 7.36 & 0.278 \\
\hline Acinetobacter & 0.3 & 0.04 & 0.662 & 1.17 & 0.21 & 0.306 \\
\hline Lactobacillus & 0.52 & 0.05 & 0.051 & 0.13 & 3.54 & 0.043 \\
\hline Clostridium & 0.12 & 0.16 & 0.636 & 0.05 & 1.71 & 0.378 \\
\hline
\end{tabular}

* unspecified to a specific genus within this family. 


\subsection{Comparison of Calprotectin Levels between Groups}

We compared fecal calprotectin levels in groups I, II, and III and its distribution is presented in Figure 3. The fecal calprotectin level in group III (median and IQR, 199.0 and 34.3-658.3 mg/kg) was significantly higher than those in group I and group II (15.0, 15.0-39.5 and 23.0, 15.0-57.0 mg/kg, respectively, $p<0.001$ for both). There was no significant difference between group I and group II $(p=0.256)$. The fecal calprotectin levels were not significantly different between individuals with non-toxigenic and toxigenic C. difficile in both group II and group III ( $p=0.496$ and 0.273 , respectively).
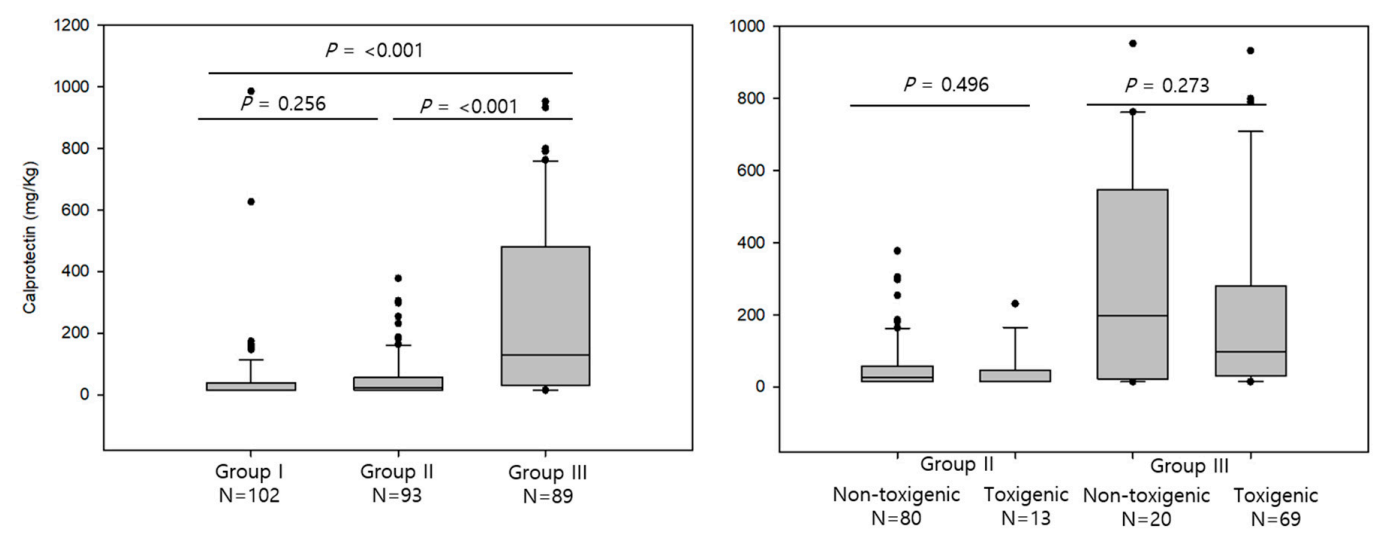

Figure 3. Fecal calprotectin levels in group I (C. difficile non-colonized), group II (C. difficile colonized), and group III (diarrhea with $C$. difficile) are shown in left and the comparisons between non-toxigenic and toxigenic C. difficile within group II and group III are shown in right. Each $p$-value (I vs. III, I vs. II, II vs. III and non-toxigenic vs. toxigenic C. difficile) was presented. The box plot shows the median and interquartile values. The whiskers indicate 1.5 times the interquartile range above and below the 75 th and 25 th percentiles. The circles indicate the outliers.

\section{Discussion}

The asymptomatic colonization rate of toxigenic $C$. difficile among healthy individuals ranges from 1.8 to $15 \%$ [14,21,22]. However, most studies were based on point prevalence detection and methodology and the target population was variable. In our study, C. difficile colonization among the screened population were $11.4 \%$ and the toxigenic portion among colonized $C$. difficile was relatively low $(14 \%)$, which was lower than our previous studies performed in the same institution using the same method [23]. This difference might be due to different populations and different criteria in this study, which only included adult, non-obese individuals among visitors for a general health examination. The toxigenic proportion was much higher in hospitalized patients with diarrhea [23].

As expected, the alpha diversity index (Chao1), as shown in Figure 1, was significantly lower in group III (diarrhea with C. difficile) compared with those in group I (C. difficile non-colonized) and group II (C. difficile colonized). Previous studies also showed decreased alpha diversity in CDI or antibiotic exposed group compared with the control [8,24]. As decreased species diversity could occur in many hospitalized patients without CDI, this feature might not be specific for CDI and could be a common feature in hospitalized patients with antibiotic exposure. The development of overt CDI needs additional host factors, such as immunity, age, or hospital stay $[3,4,25]$. In this study, the alpha diversity index between group I and group II did not significantly differ. Thus, the C. difficile colonization itself in healthy individuals does not seem to affect species diversity. The present study also showed that the alpha diversity in group II and group III was not affected by toxigenic status.

In PCoA analysis, group III clustered separately from group I and II by unweighted and weighted UniFrac analysis, which was consistent with previous studies $[8,24]$. This indicates that hospitalized patients with diarrhea have different beta diversity to healthy individuals. We could find a significant difference between group I and II by weighed UniFrac analysis. Thus, although the total species 
diversity did not change in $C$. difficile colonization (based on alpha diversity), the composition and abundance of microbiota were changed in C. difficile colonization. Regarding toxin status, subjects with non-toxigenic and toxigenic $C$. difficile clustered separately both in unweighted and weighted UniFrac matrix, indicating that the intestinal microbiota is further changed distinctly upon colonization of toxigenic $C$. difficile strains compared to non-toxigenic strains. In patients of group III, non-toxigenic and toxigenic $C$. difficile also clustered separately, but significant difference was observed only in the unweighted UniFrac matrix. Disruption of intestinal microbiota composition occurs in many hospitalized patients, which could weaken the difference between those colonized with non-toxigenic and toxigenic strains.

Our study showed different patterns of the composition of fecal microbiota in patients with diarrhea, as shown previously [8,26]. Specifically, Group III patients showed lower and higher abundance of Bacteroidetes and Proteobacteria, respectively, at the phylum level, and lower relative abundance of many genera, including butyrate-producing organisms and health-promoting bacteria $[9,27]$. Increased Parabacteroides or Enterococcus, which reflect a blooming phenomenon by reduced ecological niche competition $[7,8,28,29]$, were also observed in group III which included various hospitalized patients. These features were noted similarly in non-toxigenic and toxigenic $C$. difficile, suggesting again that this disruption of microbiota was not specific to patients with toxigenic C. difficile.

There is very limited data on the composition of intestinal microbiota in $C$. difficile colonization and a few studies only described a very limited number of subjects $[8,14,30,31]$. In this study, we could find several distinct features in C. difficile colonization, including those due to the toxigenic status of the strains. Group II (C. difficile colonized) did not show significant differences compared with non-colonized subjects (group I) at the phylum level and the results at phylum levels were the sum of the changes of many genera. At the genus level, group II showed a significantly lower abundance of many genera and a higher abundance of Enterobacteriaceae compared to group I. This pattern of change was partly similar to that observed in CDI or hospitalized patients, indicating that the genus compositions of $C$. difficile colonized subjects lies between non-colonized and hospitalized patients. Moreover, toxigenic $C$. difficile-colonized subjects showed significant changes, such as a lower abundance of Prevotella, Phascolarctobacterium, Succinivibrio, Blautia, and an unassigned genus of Ruminococcaceae and higher abundance of Bacteroides and an unassigned genus of Enterobacteriaceae than non-toxigenic $C$. difficile-colonized subjects. These features were closer to the patterns observed in hospitalized patients with diarrhea. We could not find a significant difference in hospitalized patients with diarrhea based on the presence or absence of toxigenic C. difficile.

Fecal calprotectin is a $36.5 \mathrm{kDa}$ molecule derived from the cytoplasm of neutrophils, mononuclear cells, and squamous epithelial cells and reflects the inflammatory status of the intestine [32]. The proposed clinical utility of this marker has been focused on inflammatory bowel disease (IBD) [33]. In CDI, fecal calprotectin has been shown to reflect severity $[15,16,34]$; however, this marker showed a non-specific increase in various inflammatory conditions of the intestines, thereby limiting its diagnostic utility $[35,36]$. In this study, we showed that $C$. difficile-colonized subjects, regardless of toxin status, had comparable fecal calprotectin levels to those of non-colonized subjects and significantly lower levels than in diarrhea patients with $C$. difficile. The $C$. difficile-colonized subjects seemed to show a non-inflammatory status, although they showed changes in the composition of intestinal microbiota (beta diversity). The diarrhea patients had significantly higher fecal calprotectin levels, reflecting in high inflammatory status, and the toxin status did not show significant differences in calprotectin levels, indicating the non-specific increase of this marker, as suggested by previous studies [34-36].

This study had several limitations. The cause-effect relationship between specific alterations of the microbiota and clinical status or colonization could not be elucidated in this study design. Many covariates such as age or food intake habit could also affect the intestinal microbiota composition [37]. The median age of group III was significantly higher than the other groups and old age itself could result in decreased diversity and change composition of gut microbiota in group III [7,37]. The old aged subjects were rare in general health examination and enrollment of elderly subjects was difficult. 
Several studies recently showed data regarding differences in the gut microbiota between sexes [38,39]. In this study, we could not evaluate data based on sex differences and could be further studied. In addition, the results of the OTU assignment could be different between algorithms or programs used for OTU analysis $[37,40]$.

\section{Conclusions}

There were significant alterations in the alpha and beta diversity in intestinal microbiota in hospitalized patients with diarrhea, but these alterations were not significantly affected by the toxin status of $C$. difficile. We found significant differences in the composition of intestinal microbiota in apparently healthy $C$. difficile colonized subjects in comparison to $C$. difficile non-colonized subjects. Despite the significant difference in C. difficile colonization such as lower levels of Sutterella, Blautia, Ruminococcus, Faecalibacterium, Bilophila, and Ruminococcaceae and higher levels of Enterobacteriaceae, inflammatory status and alpha diversity were not significantly different between $C$. difficile colonized and non-colonized subjects. Further investigation of the intestinal microbiota and immunological studies could provide some insight into the conditions that allow for colonization and protect against disease progression.

Author Contributions: H.-W.M. conceived the ideas and experimental design. J.Y. collected the samples and clinical data. J.-H.K. performed the experiment and analyzed the data. S.-H.H. and H.-W.M. did the interpretations and writing of the publication. All authors read and approved the final manuscript.

Funding: This research was supported by Basic Science Research Program through the National Research Foundation of Korea (NRF) funded by the Ministry of Science and ICT (2017R1A2B4005402).

Conflicts of Interest: The authors report no conflicts of interest.

\section{References}

1. Lawson, P.A.; Citron, D.M.; Tyrrell, K.L.; Finegold, S.M. Reclassification of Clostridium difficile as Clostridioides difficile (Hall and O'Toole 1935) Prevot 1938. Anaerobe 2016, 40, 95-99. [CrossRef]

2. Balsells, E.; Shi, T.; Leese, C.; Lyell, I.; Burrows, J.; Wiuff, C.; Campbell, H.; Kyaw, M.H.; Nair, H. Global burden of Clostridium difficile infections: A systematic review and meta-analysis. J. Glob. Health 2019, 9, 1-20. [CrossRef]

3. Bagdasarian, N.; Rao, K.; Malani, P.N. Diagnosis and treatment of Clostridium difficile in adults: A systematic review. JAMA 2015, 313, 398-408. [CrossRef] [PubMed]

4. Martin, J.S.; Monaghan, T.M.; Wilcox, M.H. Clostridium difficile infection: Epidemiology, diagnosis and understanding transmission. Nat. Rev. Gastroenterol. Hepatol. 2016, 13, 206-216. [CrossRef] [PubMed]

5. Lewis, B.B.; Buffie, C.G.; Carter, R.A.; Leiner, I.; Toussaint, N.C.; Miller, L.C.; Gobourne, A.; Ling, L.; Pamer, E.G. Loss of Microbiota-Mediated Colonization Resistance to Clostridium difficile Infection With Oral Vancomycin Compared With Metronidazole. J. Infect. Dis. 2015, 212, 1656-1665. [CrossRef] [PubMed]

6. Seekatz, A.M.; Young, V.B. Clostridium difficile and the microbiota. J. Clin. Investig. 2014, 124, 4182-4189. [CrossRef] [PubMed]

7. Milani, C.; Ticinesi, A.; Gerritsen, J.; Nouvenne, A.; Lugli, G.A.; Mancabelli, L.; Turroni, F.; Duranti, S.; Mangifesta, M.; Viappiani, A.; et al. Gut microbiota composition and Clostridium difficile infection in hospitalized elderly individuals: A metagenomic study. Sci. Rep. 2016, 6, 25945. [CrossRef]

8. Zhang, L.; Dong, D.; Jiang, C.; Li, Z.; Wang, X.; Peng, Y. Insight into alteration of gut microbiota in Clostridium difficile infection and asymptomatic C. difficile colonization. Anaerobe 2015, 34, 1-7. [CrossRef] [PubMed]

9. Antharam, V.C.; Li, E.C.; Ishmael, A.; Sharma, A.; Mai, V.; Rand, K.H.; Wang, G.P. Intestinal dysbiosis and depletion of butyrogenic bacteria in Clostridium difficile infection and nosocomial diarrhea. J. Clin. Microbiol. 2013, 51, 2884-2892. [CrossRef]

10. Isaac, S.; Scher, J.U.; Djukovic, A.; Jimenez, N.; Littman, D.R.; Abramson, S.B.; Pamer, E.G.; Ubeda, C. Shortand long-term effects of oral vancomycin on the human intestinal microbiota. J. Antimicrob. Chemother. 2016. [CrossRef]

11. Louie, T.J.; Byrne, B.; Emery, J.; Ward, L.; Krulicki, W.; Nguyen, D.; Wu, K.; Cannon, K. Differences of the Fecal Microflora with Clostridium difficile Therapies. Clin. Infect. Dis. 2015, 60 (Suppl. 2), S91-S97. [CrossRef] 
12. McDonald, L.C.; Gerding, D.N.; Johnson, S.; Bakken, J.S.; Carroll, K.C.; Coffin, S.E.; Dubberke, E.R.; Garey, K.W.; Gould, C.V.; Kelly, C.; et al. Clinical Practice Guidelines for Clostridium difficile Infection in Adults and Children: 2017 Update by the Infectious Diseases Society of America (IDSA) and Society for Healthcare Epidemiology of America (SHEA). Clin. Infect. Dis. 2018, 66, e1-e48. [CrossRef]

13. Crobach, M.J.T.; Baktash, A.; Duszenko, N.; Kuijper, E.J. Diagnostic Guidance for C. difficile Infections. Adv. Exp. Med. Biol. 2018, 1050, 27-44. [CrossRef] [PubMed]

14. Crobach, M.J.T.; Vernon, J.J.; Loo, V.G.; Kong, L.Y.; Pechine, S.; Wilcox, M.H.; Kuijper, E.J. Understanding Clostridium difficile Colonization. Clin. Microbiol. Rev. 2018, 31. [CrossRef]

15. Kim, J.; Kim, H.; Oh, H.J.; Kim, H.S.; Hwang, Y.J.; Yong, D.; Jeong, S.H.; Lee, K. Fecal Calprotectin Level Reflects the Severity of Clostridium difficile Infection. Ann. Lab. Med. 2017, 37, 53-57. [CrossRef] [PubMed]

16. Barbut, F.; Gouot, C.; Lapidus, N.; Suzon, L.; Syed-Zaidi, R.; Lalande, V.; Eckert, C. Faecal lactoferrin and calprotectin in patients with Clostridium difficile infection: A case-control study. Eur. J. Clin. Microbiol. Infect. Dis. 2017, 36, 2423-2430. [CrossRef] [PubMed]

17. Crobach, M.J.; Planche, T.; Eckert, C.; Barbut, F.; Terveer, E.M.; Dekkers, O.M.; Wilcox, M.H.; Kuijper, E.J. European Society of Clinical Microbiology and Infectious Diseases: Update of the diagnostic guidance document for Clostridium difficile infection. Clin. Microbiol. Infect. 2016, 22 (Suppl. 4), S63-S81. [CrossRef]

18. Cohen, S.H.; Gerding, D.N.; Johnson, S.; Kelly, C.P.; Loo, V.G.; McDonald, L.C.; Pepin, J.; Wilcox, M.H. Clinical practice guidelines for Clostridium difficile infection in adults: 2010 update by the society for healthcare epidemiology of America (SHEA) and the infectious diseases society of America (IDSA). Infect. Control. Hosp. Epidemiol. 2010, 31, 431-455. [CrossRef]

19. Yatsunenko, T.; Rey, F.E.; Manary, M.J.; Trehan, I.; Dominguez-Bello, M.G.; Contreras, M.; Magris, M.; Hidalgo, G.; Baldassano, R.N.; Anokhin, A.P.; et al. Human gut microbiome viewed across age and geography. Nature 2012, 486, 222-227. [CrossRef] [PubMed]

20. Lozupone, C.; Knight, R. UniFrac: A new phylogenetic method for comparing microbial communities. Appl. Environ. Microbiol. 2005, 71, 8228-8235. [CrossRef]

21. Barbut, F.; Day, N.; Bouee, S.; Youssouf, A.; Grandvoinnet, L.; Lalande, V.; Couturier, J.; Eckert, C. Toxigenic Clostridium difficile carriage in general practice: Results of a laboratory-based cohort study. Clin. Microbiol. Infect. 2019, 25, 588-594. [CrossRef] [PubMed]

22. Meltzer, E.; Smollan, G.; Huppert, A.; Fluss, R.; Tal, I.; Gilboa, M.; Zilberman-Daniels, T.; Keller, N.; Rahav, G.; Regev-Yochay, G.; et al. Universal screening for Clostridioides difficile in a tertiary hospital: Risk factors for carriage and clinical disease. Clin. Microbiol. Infect. 2019, 25, 1127-1132. [CrossRef] [PubMed]

23. Kim, H.N.; Kim, H.; Moon, H.W.; Hur, M.; Yun, Y.M. Toxin positivity and tcdB gene load in broad-spectrum Clostridium difficile infection. Infection 2018, 46, 113-117. [CrossRef]

24. Han, S.H.; Yi, J.; Kim, J.H.; Lee, S.; Moon, H.W. Composition of gut microbiota in patients with toxigenic Clostridioides (Clostridium) difficile: Comparison between subgroups according to clinical criteria and toxin gene load. PLoS ONE 2019, 14, e0212626. [CrossRef]

25. Moon, H.W.; Kim, H.N.; Hur, M.; Shim, H.S.; Kim, H.; Yun, Y.M. Comparison of Diagnostic Algorithms for Detecting Toxigenic Clostridium difficile in Routine Practice at a Tertiary Referral Hospital in Korea. PLoS ONE 2016, 11, e0161139. [CrossRef]

26. Buffie, C.G.; Bucci, V.; Stein, R.R.; McKenney, P.T.; Ling, L.; Gobourne, A.; No, D.; Liu, H.; Kinnebrew, M.; Viale, A.; et al. Precision microbiome reconstitution restores bile acid mediated resistance to Clostridium difficile. Nature 2015, 517, 205-208. [CrossRef]

27. Miquel, S.; Martin, R.; Rossi, O.; Bermudez-Humaran, L.G.; Chatel, J.M.; Sokol, H.; Thomas, M.; Wells, J.M.; Langella, P. Faecalibacterium prausnitzii and human intestinal health. Curr. Opin. Microbiol. 2013, 16, 255-261. [CrossRef]

28. Theriot, C.M.; Young, V.B. Interactions between the Gastrointestinal Microbiome and Clostridium difficile. Annu. Rev. Microbiol. 2015, 69, 445-461. [CrossRef]

29. Stecher, B.; Maier, L.; Hardt, W.D. 'Blooming' in the gut: How dysbiosis might contribute to pathogen evolution. Nat. Rev. Microbiol. 2013, 11, 277-284. [CrossRef]

30. Vincent, C.; Miller, M.A.; Edens, T.J.; Mehrotra, S.; Dewar, K.; Manges, A.R. Bloom and bust: Intestinal microbiota dynamics in response to hospital exposures and Clostridium difficile colonization or infection. Microbiome 2016, 4, 12. [CrossRef] [PubMed] 
31. Crobach, M.J.T.; Ducarmon, Q.R.; Terveer, E.M.; Harmanus, C.; Sanders, I.; Verduin, K.M.; Kuijper, E.J.; Zwittink, R.D. The Bacterial Gut Microbiota of Adult Patients Infected, Colonized or Noncolonized by Clostridioides difficile. Microorganisms 2020, 8, 677. [CrossRef] [PubMed]

32. Steinbakk, M.; Naess-Andresen, C.F.; Lingaas, E.; Dale, I.; Brandtzaeg, P.; Fagerhol, M.K. Antimicrobial actions of calcium binding leucocyte L1 protein, calprotectin. Lancet 1990, 336, 763-765. [CrossRef]

33. Caccaro, R.; D'Inca, R.; Sturniolo, G.C. Clinical utility of calprotectin and lactoferrin as markers of inflammation in patients with inflammatory bowel disease. Expert Rev. Clin. Immunol. 2010, 6, 551-558. [CrossRef] [PubMed]

34. Swale, A.; Miyajima, F.; Roberts, P.; Hall, A.; Little, M.; Beadsworth, M.B.; Beeching, N.J.; Kolamunnage-Dona, R.; Parry, C.M.; Pirmohamed, M. Calprotectin and lactoferrin faecal levels in patients with Clostridium difficile infection (CDI): A prospective cohort study. PLoS ONE 2014, 9, e106118. [CrossRef]

35. Manceau, H.; Chicha-Cattoir, V.; Puy, H.; Peoc'h, K. Fecal calprotectin in inflammatory bowel diseases: Update and perspectives. Clin. Chem. Lab. Med. 2017, 55, 474-483. [CrossRef]

36. Sipponen, T.; Kolho, K.L. Fecal calprotectin in diagnosis and clinical assessment of inflammatory bowel disease. Scand. J. Gastroenterol. 2014, 50, 74-80. [CrossRef]

37. Goodrich, J.K.; Di Rienzi, S.C.; Poole, A.C.; Koren, O.; Walters, W.A.; Caporaso, J.G.; Knight, R.; Ley, R.E. Conducting a microbiome study. Cell 2014, 158, 250-262. [CrossRef] [PubMed]

38. Org, E.; Mehrabian, M.; Parks, B.W.; Shipkova, P.; Liu, X.; Drake, T.A.; Lusis, A.J. Sex differences and hormonal effects on gut microbiota composition in mice. Gut Microbes 2016, 7, 313-322. [CrossRef] [PubMed]

39. Takagi, T.; Naito, Y.; Inoue, R.; Kashiwagi, S.; Uchiyama, K.; Mizushima, K.; Tsuchiya, S.; Dohi, O.; Yoshida, N.; Kamada, K.; et al. Differences in gut microbiota associated with age, sex, and stool consistency in healthy Japanese subjects. J. Gastroenterol. 2019, 54, 53-63. [CrossRef]

40. Werner, J.J.; Koren, O.; Hugenholtz, P.; DeSantis, T.Z.; Walters, W.A.; Caporaso, J.G.; Angenent, L.T.; Knight, R.; Ley, R.E. Impact of training sets on classification of high-throughput bacterial 16s rRNA gene surveys. ISME J. 2012, 6, 94-103. [CrossRef]

(C) 2020 by the authors. Licensee MDPI, Basel, Switzerland. This article is an open access article distributed under the terms and conditions of the Creative Commons Attribution (CC BY) license (http://creativecommons.org/licenses/by/4.0/). 